\title{
A saúde mental dos idosos em tempos de pandemia: uma revisão de literatura
}

\author{
Mental health of the elderly in times of pandemic: a literature review
}

Salud mental de los ancianos en tiempos de pandemia: revisión de la literatura

Camila Abreu Pinto Cunha ${ }^{*}$, Bianca da Rocha Siqueira1', Marianna Ramalho de Sousa ${ }^{1}$, Hélcio Serpa de Figueiredo Júnior ${ }^{1}$.

\section{RESUMO}

Objetivo: Analisar na literatura acerca da saúde mental dos idosos em tempos de pandemia. Revisão bibliográfica: $O$ envelhecimento acarreta mudanças fisiológicas quem culminam no aumento da suscetibilidade às doenças que podem levar ao comprometimento das capacidades cognitivas e físicas. Nesse contexto, o isolamento social necessário para o controle da pandemia juntamente com o estresse, o luto, o medo da morte e do adoecimento, as perdas financeiras somados aos desafios inerentes do envelhecimento são fatores de risco para o adoecimento mental dos idosos em tempos de pandemia. Durante a pandemia da COVID-19, a composição domiciliar dos idosos um risco que independe da conformação. Idosos que habitam sozinhos morando sozinhos estão mais expostos à solidão. Já aqueles que residem com outras pessoas correm mais risco de serem contagiados. Os transtornos mais comuns, nesse contexto, são a depressão e os quadros ansiosos que podem levar ao suicídio. Considerações finais: Nesse sentido, torna-se essencial a integração social do idoso através do meio digital, a diminuição da exposição destes indivíduos às notícias da pandemia e o incentivo à pratica de atividades físicas mesmo que em domicílio e de maneira remota a fim de mitigar os efeitos psicológicos da pandemia nos idosos.

Palavras-chave: Saúde mental, Pandemia, Idosos.

\begin{abstract}
Objective: To analyze the literature on the mental health of the elderly in times of a pandemic. Bibliographic review: Aging causes physiological changes that culminate in increased susceptibility to diseases that can lead to impairment of cognitive and physical abilities. In this context, the social isolation necessary to control the pandemic together with stress, grief, fear of death and illness, financial losses added to the inherent challenges of aging are risk factors for the mental illness of the elderly in times of crisis. pandemic. During the COVID-19 pandemic, the household composition of the elderly is a risk that is independent of conformation. Elderly people who live alone are more exposed to loneliness. Those who live with other people are more at risk of being infected. The most common disorders in this context are depression and anxiety that can lead to suicide. Final considerations: In this sense, it is essential to socially integrate the elderly through the digital environment, to reduce the exposure of these individuals to the news of the pandemic and to encourage the practice of physical activities even at home and remotely in order to mitigate the psychological effects of the pandemic on the elderly.
\end{abstract}

Key words: Mental health, Pandemic, Aged.

\section{RESUMEN}

Objetivo: Analizar la literatura sobre la salud mental de los ancianos en tiempos de pandemia. Revisión bibliográfica: El envejecimiento provoca cambios fisiológicos que culminan en una mayor susceptibilidad a enfermedades que pueden conducir al deterioro de las capacidades cognitivas y físicas. En este contexto, el aislamiento social necesario para controlar la pandemia junto con el estrés, el duelo, el miedo a la muerte y la enfermedad, las pérdidas económicas sumadas a los desafíos inherentes al envejecimiento son factores de riesgo para la enfermedad mental de los adultos mayores en tiempos de crisis pandémica. Durante la pandemia de COVID-19, la composición del hogar de los ancianos es un riesgo independiente de la conformación. Las personas mayores que viven solas están más expuestas a la soledad. Quienes viven con

${ }^{1}$ Universidade de Vassouras (UV), Vassouras - RJ. *E-mail: camilaabreupc@hotmail.com

SUBMETIDO EM: 1/2022

ACEITO EM: 1/2022

PUBLICADO EM: 2/2022 
otras personas corren más riesgo de infectarse. Los trastornos más comunes en este contexto son la depresión y la ansiedad que pueden llevar al suicidio. Consideraciones finales: En este sentido, es fundamental integrar socialmente a las personas mayores a través del entorno digital, reducir la exposición de estas personas a las noticias de la pandemia y fomentar la práctica de actividades físicas incluso en casa y a distancia para mitigar los efectos psicológicos de la pandemia en las personas mayores.

Palabras clave: Salud mental, Pandemia, Ancianos.

\section{INTRODUÇÃO}

A doença causada pelo novo coronavírus (Covid-19) surgiu na China em dezembro de 2019, tratando-se de uma patologia infecciosa que causa uma síndrome respiratória aguda grave (SANTOS SS, et al., 2020). Em março de 2020, após a detecção de casos da doença em 114 países, devido à facilidade de transmissão do vírus e do aumento exponencial do número de contaminados, a Organização Mundial da Saúde (OMS) declarou a situação global de disseminação da COVID-19 como uma pandemia (JúNIOR FEN, et al., 2020; MONTEIRO IVL, et al., 2021).

Diante da acelerada disseminação do vírus foram instituídas medidas de distanciamento e isolamento social. Em epidemias, as medidas de isolamento estão associadas ao aumento de transtornos mentais, como depressão e ansiedade, seja pela exacerbação de distúrbios já existentes ou pela inabilidade para o enfrentamento da situação inerente ao contexto da doença (PECOITS RV, et al., 2021; ZWIELEWSKI G, et al., 2020).

Nesse contexto, o principal grupo afetado foi o dos idosos (PECOITS RV, et al., 2021). Desde o inicio da pandemia, esta população se tornou a mais vulnerável ao vírus devido à presença de comorbidades como diabetes, hipertensão, doenças respiratórias e cardiopatias o que por si só acarretou estresse nesses indivíduos devido ao medo de adoecimento (PEREIRA-ÁVILA FMV, et al., 2021). A pandemia além de representar enorme ameaça à vida, pode impor aos idosos maior risco de pobreza, trauma de estigma, perda de suporte social, discriminação e isolamento social (ROMERO DE, et al., 2021).

Soma-se a isso, o isolamento social e a solidão que já afetavam uma porção significativa dos idosos antes da pandemia foram agravados, especialmente para aqueles que residem sozinhos (PECOITS RV, et al., 2021). A tristeza é o sentimento que mais que mais contribui para a solidão e se relaciona, em um contexto de pandemia, com o luto coletivo, alta mortalidade de sua faixa etária e falta de políticas públicas de proteção social (ROMERO DE, et al., 2021).

Em decorrência da pandemia e do seu grande impacto na vida da população, principalmente idosa é essencial o estudo da influência deste acontecimento na saúde mental. O objetivo do estudo foi analisar na literatura acerca da saúde mental dos idosos em tempos de pandemia.

\section{REVISÃO BIBLIOGRÁFICA}

\section{O envelhecimento e fatores associados a transtornos mentais na população idosa na pandemia}

O envelhecimento acarreta mudanças fisiológicas quem culminam no aumento da suscetibilidade à doenças que podem levar ao comprometimento das capacidades cognitivas e físicas. Fato que leva ao declínio da cognição, da mobilidade e restrição nas atividades de vida, determinando declínios de capacidade funcional e de respostas adaptativas que são indispensáveis para a realização das atividades da vida diária. Isso impacta diretamente na saúde mental desses indivíduos que antes ativos, passam a depender de outras pessoas para realização de atividades cotidianas (BEZERRA GKSD, et al., 2021).

O número de idosos vem crescendo rapidamente no mundo devido a transição demográfica com a redução das taxas de fecundidade e increment da expectativa de vida da população. No Brasil, mais de 28 milhões de indivíduos na faixa etária acima de 60 anos, o que representa $13 \%$ da população do país passando para quase 70 milhões em 2050 (SILVA ML, et al.,2020; MONTEIRO IVL, et al., 2021). 
A pandemia coincide com o envelhecimento da população, o qual é considerado o principal evento demográfico do século XXI a nível mundial e nacional (ROMERO DE, et al., 2021). Os idosos são a população mais vulnerável a COVID-19 pela maior imunossenescência e associação com comorbidades prévias, principalmente naqueles com doenças crônicas como diabetes e hipertensão. A maior taxa de mortalidade ocorre entre os indivíduos com 80 anos ou mais, em que 14,8\% dos infectados morreram, comparado a $8,0 \%$ entre os idosos de 70 a 79 anos e $8,8 \%$ entre aqueles de 60 a 69 anos (HAMMERSCHMIDT KSA e SANTANA RF, 2020).

O medo eleva os níveis de ansiedade e estresse em pessoas saudáveis e exacerba os sintomas daqueles com transtornos psiquiátricos pré-existentes, com isso durante epidemias e pandemias, o número de pessoas cuja saúde mental é afetada tende a ser maior que o número de pessoas afetadas pela infecção em si (SILVA ML, et al., 2020).

O isolamento social oriundo da pandemia não é o único fator estressor presente no desenvolvimento de transtornos emocionais. Também é importante ressaltar os sentimentos negativos oriundos da pandemia em si, e não apenas dos aspectos que tentam reduzir seus efeitos; ou seja, o medo e ansiedade da perda de familiares e da própria vida, haja vista a possibilidade de infecção, de modo que os idosos vivem num estado de luto antecipado, sofrendo também pela possibilidade de perda diante da tristeza e incerteza inerentes à conjuntura de pandemia e isolamento (LOSADA-BALTAR A, et al., 2020; OLIVEIRA VV, et al., 2021).

O estresse da diminuição da condição financeira em um momento de recessão econômica, pobreza e desemprego constitui um risco psicossocial muito comum. Assim, são fatores que podem afetar negativamente a saúde mental de um indivíduo durante a pandemia (OLIVEIRA JT, et al., 2021).

Nos países subdesenvolvidos, nos quais vivem $69 \%$ da população mundial com 60 anos ou mais e cujos sistemas de saúde são mais frágeis, a pandemia de COVID-19 pode vir a ter um impacto maior. No Brasil, $43 \%$ dos idosos são a principal fonte de renda nos lares do país, além de representarem $30 \%$ dos analfabetos e $22,5 \%$ daqueles que não tem acesso à internet. As condições socioeconômicas dos idosos os colocam em uma posição mais vulnerável por afetarem diretamente em sua qualidade de vida, tornando-os mais propensos a quadros de ansiedade e depressão. Tais questões podem se intensificadas no contexto de pandemia (JÚNIOR FEN, et al., 2020).

Durante a pandemia da COVID-19, a composição do domicílio do idoso representa risco de forma independente da sua conformação. Idosos que habitam sozinhos podem depender de ajuda para adquirir alimentos, suporte afetivo, econômico, cuidados à saúde e outros, estando mais expostos à solidão. Já aqueles que residem com outros familiars correm risco de serem infectados por integrantes da residência que mantém contato com o meio externo, sendo um fator de estresse para todos os residentes da casa (ROMERO DE, et al., 2021).

Alguns estudos apontam para o incremento da solidão na população idosa durante a pandemia. $O$ aumento da angústia e da solidão pode ser suficiente para agravar clinicamente a saúde mental entre adultos mais velhos, estando associada a vários desfechos negativos, tais quais depressão, ansiedade e aumento na morbimortalidade (STOLZ E, et al., 2021; SHIRA A, et al., 2020). Um estudo demonstrou que $24 \%$ dos idosos foram classificados como socialmente isolados, e $43 \%$ dos individuos com mais de 60 anos relataram solidão subjetiva (TYRREL CJ e WILLIAMS KN, 2020). A solidão é considerada um fator de risco para o aumento dos sintomas depressivos em idosos, além de ser considerada fator preditivo de risco para o declínio cognitivo e redução da funcionalidade geral. Dessa forma, a solidão pode piorar tanto a saúde física quanto mental do idoso (STOLZ E, et al., 2021; SHIRA A, et al., 2020).

Outro estudo realizado em Hong Kong com 583 pacientes de clinicas públicas de atenção primária mensurou resultados comparativos da saúde mental de idosos nos períodos pré e pós pandemia. 0 trabalho mostrou que os indivíduos mais velhos com multimorbidades experimentavam uma pior saúde psicossocial, com um incremento da quantidade de consultas médicas para doenças crônicas após a pandemia. Ademais, identificaram que ser mulher, morar sozinha e ter mais doenças crônicas está 
associado com um maior risco de desfechos negativos. Outro fator constatado foi que os idosos que moravam sozinhos tinham maiores chances de solidão durante a pandemia, pois mesmo aqueles que tinham auxílio de pessoas que não residiam com eles para suas atividades tiveram redução nessa colaboração devido a pandemia, agravando a solidão e suas consequências (SANTOS RG, et al., 2021).

Cita-se, ainda, a grande exposição a informações acerca da grande quantidade de mortos e infectados o que pode ser um potencializador no estresse e elevar o desenvolvimento de transtornos mentais. Tal exposição a notícias frequentes de uma situação como uma pandemia é conhecida por ser prejudicial à saúde mental. A exposição indireta ao trauma em massa pela mídia pode incrementar a taxa de sintomas de transtorno de estresse pós-traumático (OLIVEIRA JT, et al., 2021).

Um estudo com a população idosa na China, durante a pandemia de coronavírus em 2020, registrou que a exposição frequente às redes sociais e notícias sobre a COVID-19 está relacionada a um maior risco de sintomas de ansiedade e depressão, os quais persistem mesmo após outros fatores terem sido debelados (DUARTE MQ, et al., 2020).

\section{Depressão em idosos na pandemia}

A doença crônica mais prevalente na velhice é a depressão, que está diretamente interligada com o aumento da morbimortalidade, ao déficit de autocuidado e a baixa adesão de tratamentos sendo caracterizada como a presença de humor triste, vazio ou irritável, acompanhado de alterações somáticas e cognitivas. Estas alterações, inevitavelmente, afetam áreas importantes na vida dos indivíduos. As alterações decorrentes do processo de envelhecimento podem dificultar o diagnóstico e o tratamento da depressão, uma vez que nesta fase da vida, podem surgir patologias crónicas, retardo psicomotor, sintomas subjetivos de perda da concentração e da memória e alterações do sono (AGUIAR MS, et al., 2021).

Os principais fatores relacionados com uma saúde desfavorável e uma má qualidade de vida são a presença de transtornos de ansiedade e depressão, isolamento social, falta de exercício e dependência de atividades básicas diárias (como cuidados pessoais) e instrumentais (AGUIAR MS, et al., 2021).

Um estudo realizado com participantes de todas as regiões do Brasil de maneira online indicou que os principais fatores associados à depressão na pandemia do COVID-19 foram estado civil. sexo, escolaridade e renda. As mulheres idosas que têm ocupação com na qual há exposição ao COVID-19 apresentaram maiores escores de depressão. Não ter graduação e ter idade igual ou maior a 85 anos esteve associado à maior frequência de sintomas depressivos; por outro lado, receber cinco salários, ou mais, diminuiu as chances dessa sintomatologia (PEREIRA-ÁVILA FMV, et al., 2021; CIGILOGLU A, et al., 2021).

Após o estabelecimento das medidas de distanciamento social houve uma mudança negativa no humor, nas conexões sociais e uma diminuição significativa na qualidade de vida dos idosos. Nesse contexto características como "ser positivo" e "ter apoio de amigos" impactaram de forma positiva na maneira de lidar com a pandemia e resultaram em menos depressão em idosos com essas características. Já a comunicação menos frequente e a solidão percebida se relacionaram à maior prevalência de sintomas depressivos (MISTRY SK, et al., 2021; PECOITS RV, et al., 2021).

Outro fator que é capaz de aumentar a incidência de sintomas depressivos foram condições médicas preexistentes, que elevam o risco na apresentação de sintomas depressivos em até 91\% (MISTRY SK, et al., 2021; PECOITS RV, et al., 2021).

\section{Ansiedade em idosos na pandemia}

Estados de ansiedade podem ser considerados inerentes ao organismo e possuem valor de sobrevivência, já que podem sinalizar para situações onde há um perigo eminente. Todavia, os estados de ansiedade podem se tornar um problema quando se tornam desproporcionais, isto é, quando acontece com frequência, intensidade ou duração suficiente para limitar as ações do sujeito no ambiente. Desse modo, deve-se considerar que, para um idoso em contexto de isolamento social, estados ansiosos podem ocorrer e serem adaptativos a tal situação e outros quadros de ansiedade podem acabar gerando algum tipo de problema ou sofrimento (JÚNIOR FEN, et al., 2020). 
O isolamento social impactou diretamente no convívio social dos idosos, já que os impossibilitou de realizar contato com outros indivíduos, devido a um menor acesso às tecnologias de comunicação, o que levou uma maior propensão a desenvolver quadros de ansiedade. Associado ao isolamento social, o medo do adoecimento e da morte tanto para si quanto de entes próximos em decorrência da pandemia, induziu a existência de alguns transtornos de ansiedade, como ataques de pânico, insônia e estresse pós- traumático (PECOITS RV, et al., 2021).

Ademais, além da preocupação do idoso em contrair o coronavírus, os sintomas de ansiedade também podem se correlacionar com o medo de uma quarentena com isolamento por um longo period de tempo, já que os problemas médicos crônicos e quadros álgicos não tratados de forma efetiva, em decorrência das novas regras de funcionamento dos hospitais durante a pandemia, podem aumentar alterações negativas na qualidade de vida, o que pode impactar ainda mais os sintomas emocionais em relação à doença subjacente (PECOITS RV, et al., 2021).

Em um estudo que buscou analisar a influência da desconexão social e da percepção do isolamento social sobre os quadros de depressão e ansiedade, analisou-se dados de 3005 sujeitos com idades entre 57 e 85 anos. A desconexão social é um tipo de isolamento social caracterizado pela escassez de contato com outras pessoas e pela falta de atividades ou participação em grupos. Nesse contexto, a percepção do isolamento social se refere ao que é sentido pelo sujeito ao se identificar nessa situação, por exemplo, sentimentos de solidão e falta de apoio. Os resultados indicaram que tanto a desconexão social, quanto a percepção do isolamento social são diretamente proporcionais às taxas de depressão e ansiedade. Concluise que a desconexão social e a percepção do isolamento social estão diretamente relacionadas a manifestação de quadros depressivos e ansiosos (SANTIN ZI, et al., 2020; JÚNIOR FEN, et al., 2020).

\section{Suicídio}

A depressão é o maior fator de risco para suicídio na população idosa. Em decorrência do isolamento social em idosos, houve uma potencialização da angústia e do medo por estar distante de seus entes queridos considerando o avanço da idade e que não há um prazo para o fim do isolamento, e ainda, o processo de envelhecimento traz consigo sentimentos de solidão que podem ser intensificados durante 0 distanciamento social causado pela pandemia do coronavírus. Na pandemia muitos idosos apresentam dificuldades ao vivenciar situações de desespero, ruptura dos vínculos afetivos desencadeando tristeza, angústia e solidão. A vulnerabilidade emocional para aqueles que residem sozinhos pode ser maior, podendo evoluir para estados depressivos cujo desfecho pode ser a ideação suicida, tentativa ou o próprio ato de suicídio (REIS EM, et al., 2021).

A pandemia da Covid-19 mostrou que as medidas de isolamento, solidão e depressão podem levar a consequências mais graves tais quais o suicídio. Um estudo na Índia demonstrou que os idosos que sofrem com transtornos neuropsiquiátrico estão mais sujeitos ao desenvolvimento, durante o período de pandemia, de sintomatologia depressiva e de ansiedade, o que pode levar a um risco aumentado de suicídio nessa população. Além disso, outro estudo revelou que o estresse, sintoma exacerbado pela pandemia, aumenta a gravidade dos sintomas em indivíduos com transtornos psiquiátricos preexistentes, o que pode culminar em desfechos fatais para o idoso acometido por essas patologias (JÚNIOR FEN, et al., 2020).

\section{Prevenção dos transtornos mentais em idosos no contexto de pandemia}

O medo de se contaminar e do adoecimento e falecimento de pessoas próximas pode desencadear estados de preocupação e ansiedade, o que em idosos pode ser ainda mais intenso devido à alta mortalidade desse grupo. Nesse sentido, pode-se buscar reduzir o contato com a alta densidade de informação disponível sobre o COVID-19 disponível nas mídias digitais e nos telejornais de modo a evitar gastar tempo excessivo com a exposição a informações em detrimento de atividades reforçadoras positivas, tais quais o consumo de outras mídias como músicas, filmes e séries (JÚNIOR FEN, et al., 2020; PECOITS $\mathrm{RV}$, et al., 2021).

É importante que os idosos sejam ensinados e aprendam a selecionar e priorizar as notícias relevantes e a reconhecer as fake news. Notícias falsas, comumente, são conteúdos apelativos e alarmantes 
relacionados à saúde e, por isso, podem produzir estados de ansiedade nessa parcela da população sem habilidades para avaliar a qualidade das informações acessadas (JÚNIOR FEN, et al., 2020).

Com a pandemia houve a necessidade de comunicação, integração social e busca de informações de maneira segura de modo a diminuir a cadeia de contaminação. Nesta ótica, uma das estratégias encontradas foi a utilização da tecnologia digital, empregada para estreitar o distanciamento social, principalmente entre os idosos. A inclusão digital desse grupo e o uso da Internet propicia melhor contato social e familiar, além destes manifestarem satisfação com o aprendizado e com as oportunidades que a inclusão digital proporciona nas atividades diárias, no entretenimento, na preservação da saúde mental, na motivação para aprender o novo, na melhoria da memória e no rompimento de obstáculos que são impostos pela sociedade (COSTA DES, et al., 2021).

Adultos mais velhos com acesso ao ensino digital possuem melhor performance cognitiva, linguística e redução dos sintomas de depressão, provando que a tecnologia exerce efeito positivo sobre a saúde mental do idosos. A inclusão digital reduz a sensação de isolamento, reduz a solidão, aumenta a independência e prazer, aumenta a sensação de segurança, possibilita um bem-estar psicológico e melhora a satisfação com a vida e mantém os isolados conectados através da manutenção do contato social (COSTA DES, et al., 2021).

Pode-se citar ainda, a importância da atividade física é como apoio fundamental na saúde física e mental, além de promover efeitos adicionais positivos no envelhecimento e em doenças associadas ao processo. O bem-estar psicológico pode ser conquistado e mantido no período de pandemia através do estímulo à prática de exercícios físicos que estimulam a coordenação motora e a memória. Isso pode ainda estar conjugado a inserção do idoso no meio digital, através da pratica de atividades físicas por meio de plataformas digitais ou exergames, um tipo de jogo de videogame não sedentário, o qual requer esforço físico do participante para ser jogado (ROCHA SV, et al., 2020).

\section{CONSIDERAÇÕES FINAIS}

O distanciamento social tão necessário para contenção da pandemia impactou a saúde mental dos indivíduos, com destaque para os idosos, população mais vulnerável ao vírus e que já enfrenta os desafios inerentes ao envelhecimento. Tais desafios combinados aos sentimentos de solidão e restrição social podem ocasionar alterações de humor, levando a quadros de depressão e ansiedade no idoso. Nesse contexto, torna-se essencial a integração social do idoso através do meio digital, a diminuição da exposição destes indivíduos às notícias da pandemia e o incentivo à pratica de atividades físicas mesmo que em domicílio e de maneira remota a fim de mitigar os efeitos psicológicos da pandemia nos idosos.

\section{REFERÊNCIAS}

1. AGUIAR MS, et al. Covid-19 e seu impacto na saúde mental do idoso, uma revisão da literatura. Brazilian Journal of Health Review, 2021; 4 (2): 8270-8281.

2. BEZERRA GKSD, et al. Efeitos do isolamento social para a saúde de pessoas idosas no contexto da pandemia de Covid-19: um estudo de revisão integrativa. Research, Society and Development, 2021; 10(4): e23010414070.

3. CIGILOGLU A, et al. How have older adults reacted to coronavirus disease 2019? Psychogeriatrics, 2021; 21(1): $112-117$

4. COSTA DES, et al. A influência das tecnologias na saúde mental dos idosos em tempos de pandemia: uma revisão integrativa. Research, Society and Development, 2021; 10 (2): e8210212198.

5. DUARTE MQ, et al. COVID-19 e os impactos na saúde mental: uma amostra do Rio Grande do Sul, Brasil. Ciência \& Saúde Coletiva, 2020; 25(9): 3401-3411.

6. HAMMERSCHMIDT KSA, SANTANA RF. Saúde do idoso em tempos de pandemia COVID-19. Cogitare enferm, 2020; 25: e72849.

7. JÚNIOR FEN, et al. Ansiedade em Idosos em tempos de isolamento social no Brasil (COVID-19). Rev Bras de Análise do Comportamento, 2020; 16(1): 50-56.

8. LOSADA-BALTAR, A. et al. Differences in anxiety, sadness, loneliness and comorbid anxiety and sadness as a function of age and self-perceptions of aging during the lock-out period dueto COVID-19. Rev Esp Geriatr Gerontol, 2020; 55(5): 272-278. 
9. MISTRY SK, et al. Exploring depressive symptoms and its associates among Bangladeshi older adults amid Covid19 pandemic: findings from a cross-sectional study. Soc Psychiatry Psychiatric Epidemiol., 2021; 1-11.

10. MONTEIRO IVL, et al. Idosos e saúde mental: impactos da pandemia COVID-19. Brazilian Journal of Health Review, $2021 ; 4$ (2): 6050-6061.

11. OLIVEIRA JT, et al. Saúde Mental dos Idosos em Tempos de Pandemia em tempos de pandemia Covid-19. Revista Coleta Científica, 2021; 5 (9): 20 - 30.

12. OLIVEIRA VV, et al. Impactos do isolamento social na saúde mental de idosos durante a pandemia pela Covid-19. Brazilian Journal of Health Review, 2021; .4(1): 3718-3727.

13. PECOITS RV, et al. O impacto do isolamento social na saúde mental dos idosos durante a pandemia da Covid-19. Revista da AMRIGS, 2021; 65(1): 101-108.

14. PEREIRA-ÁVILA FMV, et al. Fatores associados aos sintomas de depressão entre idosos durante a pandemia da COVID-19. Texto Contexto Enferm, 2021; 30: e20200380.

15. REIS EM, et al. Ideação suicida e tentativa de suicídio em idosos: fatores de risco associados. Revista IberoAmericana de Humanidades, Ciências e Educação, 2021, 7(6): 211-220.

16. ROCHA SV, et al. A pandemia de COVID-19 e a saúde mental de idosos: possibilidades de atividade física por meio dos Exergames. Rev Bras Ativ Fís Saúde, 2020; 25:e0142.

17. ROMERO DE, et al. Idosos no contexto da pandemia da COVID-19 no Brasil: efeitos nas condições de saúde, renda e trabalho. Cad. Saúde Pública, 2021; 37(3): e00216620

18. SANTOS SS, et al. Isolamento social: um olhar a saúde mental de idosos durante a pandemia do COVID-19. Research, Society and Development, 2020; 9(7): e392974244.

19. SANTOS RG, et al. A saúde mental dos idosos diante o distanciamento social em tempos de COVID-19. Brazilian Journal of Development, 2021; 7 (9): 87374-87384.

20. SANTIN Zl, et al. Social disconnectedness, perceived isolation, and symptoms of depression and anxiety among older Americans (NSHAP): a longitudinal mediation analysis. The Lancet Public Health, 2020; 5(1):62-70.

21. SHIRA A, et al. Covid-19-Related Loneliness and Psychiatric Symptoms Among Older Adults: The Buffering Role of Subjective Age. Am J Geriatr Psychiatry, 2020; 28(11): 1200- 1204.

22. SILVA ML, et al. Impacto na saúde mental do idoso durante o período de isolamento social em virtude da doença COVID-19: uma revisão literária. Revista Diálogos em Saúde, 2020; 3(1): 1-16.

23. STOLZ E, et al. The impact of Covid-19 restriction measures on loneliness among older adults in Austria. Eur $\mathrm{J}$ Public Health, 2021;31(1): 44-49.

24. TYRREL CJ, WILLIAMS KN. The paradox of social distancing: Implications for older adults in the context of Covid-19. Psychol Trauma, 2020; 12(1):214-216.

25. ZWIELEWSKI G, et al. Protocolos para Tratamento psicológico em pandemias: as demandas em saúde mental produzidas pela COVID-19. Debates em Psiquiatria, 2020; 10(2): 30-7. 